\title{
Spectral analysis and slow spreading dynamics on complex networks
}

\author{
Géza Ódor \\ Research Centre for Natural Sciences, Hungarian Academy of Sciences, MTA TTK MFA, P.O. Box 49, H-1525 Budapest, Hungary
}

(Received 21 June 2013; published 5 September 2013)

\begin{abstract}
The susceptible-infected-susceptible (SIS) model is one of the simplest memoryless systems for describing information or epidemic spreading phenomena with competing creation and spontaneous annihilation reactions. The effect of quenched disorder on the dynamical behavior has recently been compared to quenched meanfield (QMF) approximations in scale-free networks. QMF can take into account topological heterogeneity and clustering effects of the activity in the steady state by spectral decomposition analysis of the adjacency matrix. Therefore, it can provide predictions on possible rare-region effects, thus on the occurrence of slow dynamics. I compare QMF results of SIS with simulations on various large dimensional graphs. In particular, I show that for Erdős-Rényi graphs this method predicts correctly the occurrence of rare-region effects. It also provides a good estimate for the epidemic threshold in case of percolating graphs. Griffiths Phases emerge if the graph is fragmented or if we apply a strong, exponentially suppressing weighting scheme on the edges. The latter model describes the connection time distributions in the face-to-face experiments. In case of a generalized Barabási-Albert type of network with aging connections, strong rare-region effects and numerical evidence for Griffiths Phase dynamics are shown. The dynamical simulation results agree well with the predictions of the spectral analysis applied for the weighted adjacency matrices.
\end{abstract}

DOI: 10.1103/PhysRevE.88.032109

PACS number(s): 05.70.Ln, 89.75.Hc, 89.75.Fb

\section{INTRODUCTION}

Dynamical processes evolving on complex networks are of current research interest $[1,2]$. In networks with a large topological dimension defined as $N \propto r^{d}$, where $N$ is the number of nodes within the (chemical) distance $r$, the dynamics is expected to be exponentially fast. However, there are observations showing the appearance of generically slow time evolution. For example, in the working memory of the brain [3] or in the recovery processes following a virus pandemic $[4,5]$ power-law types of time dependencies have been found, resembling dynamical critical phenomena [6]. In social networks the occurrence of generic slow dynamics was suggested to be the result of bursty behavior of agents connected by small world networks [7].

Another possible explanation is related to the emergence of arbitrarily large, rare regions (RR) that can change their state exponentially slowly as the function of their sizes. Near phase transitions from active to inactive states in disordered systems [8-10] a so-called Griffiths Phase (GP) [11,12] can develop, characterized by nonuniversal, power-law dynamics. Griffiths singularities affect the dynamical behavior both below and above the transition point and can be best described via renormalization group methods in networks [13-16]. It has been conjectured [17-19] that such slow dynamics can occur only in finite dimensional networks as the consequence of heterogeneity: explicit reaction or purely topological disorder. This is based on optimal fluctuation theory and simulations of the contact process (CP) [21,22] on Erdôs-Rényi (ER) [20] and on generalized small world networks [23-25]. In the case of networks with an infinite topological dimension, like the Barabási-Albert (BA) [26] graph, slow dynamics has been found only in tree networks and weighting schemes, that suppress the information propagation among hubs $[27,28]$.

The susceptible-infected-susceptible (SIS) model [29] is another fundamental system to describe simple epidemic (information) possessing binary site variables: infected(active) or healthy(inactive). Infected sites propagate the epidemic (or active) all of their neighbors with rate $\lambda$ or recover (spontaneously deactivate) with rate 1 . SIS differs from the CP in which the branching rate is normalized by $k$, the number of outgoing edges of a vertex, thus it allows an analytic treatment, using symmetric matrices. By decreasing the infection rate of the neighbors a continuous phase transition occurs at some $\lambda_{c}$ critical point from a state with finite activity density $\rho$ to an inactive steady state with $\rho=0$. The latter is also called absorbing, because no spontaneous activation of sites is allowed. In [28] it was shown that a dissortative weighing scheme of the edges $w_{i j} \in(0,1)$ can effectively slow down the information propagation in SIS model and result in long-living rare regions, causing slow dynamics.

Heterogeneous mean-field (HMF) theory represents an exact result in annealed networks and provides a good approximation in networks with high $d$, when the dynamical fluctuations are irrelevant [30,31]. To describe quenched heterogeneity of the network the so-called quenched meanfield (QMF) approximation is introduced [32-34]. In [28] I compared density decay simulation results of the SIS model with the QMF approximations. Here I show further evidences for the agreement of QMF and dynamical simulations in case of certain ER and BA graphs. In particular, I investigate the prediction of QMF for the interaction weight scheme of [35] applied for ER graphs. This kind of disorder on interactions is important, because face-to-face experiments [36] resulted in such distribution of connect intensities that can be modeled with it. In $[27,28] k$ dependent weights were applied on the edges, while in [17-19] the infection probability of nodes were reduced to slow down the fast epidemic spreading in small world networks. Now I apply QMF for $k$ independent weights, distributed and frozen on the graph edges before the start of the epidemic process. Therefore, here the occurrence of slow dynamics can be understood more naturally than in case of topology driven heterogeneity of $[27,28]$ or in the case of the generalized small world networks in [17-19]. 
In the original BA graph construction one starts from a single connected node and adds new links with the linear preferential rule. This causes initial nodes with high connectivity and those attached at step $i$ will have a vanishing average degree $\left\langle k_{i}\right\rangle \propto 1 / i^{1 / 2}$. In various network studies, like article citations [37] and model calculations [38] the degradation of connection capability of aging nodes has been analyzed. It is well known that in neural networks this happens indeed. In this paper I investigate a generalized model, in which fraction of edges of the aging nodes are removed by a random, linear preferential rule [39]. In this case the edge distribution of the BA graph $P(k) \propto k^{-3}$ will be cut off by an exponential factor for large $k$-s and the QMF suggests GP behavior in agreement with the dynamical simulations.

\section{SPECTRAL ANALYSIS AND QUENCHED MEAN-FIELD APPROXIMATIONS}

A mean-field theory of the SIS model [32,34], capable of taking into account the topological heterogeneity in a network of size $N$ is based on the rate equation of $\rho_{i}(t)$, the infection probability of node $i$ at time $t$ :

$$
\frac{d \rho_{i}(t)}{d t}=-\rho_{i}(t)+\lambda\left(1-\rho_{i}(t)\right) \sum_{j=1}^{N} A_{i j} w_{i j} \rho_{j}(t) .
$$

Here $A_{i j}$ is an element of the adjacency matrix assigned with 1 , if there is an edge between nodes $i$ and $j$ or 0 otherwise and $w_{i j}$ describes the possibility of weights attributed to the edges. For large times the SIS model evolves into a steady state, with an order parameter $\rho \equiv\left\langle\rho_{i}\right\rangle$. This equation with symmetric weights under the exchange of $i \leftrightarrow j$ can be treated by a spectral decomposition (SD) on an orthonormal eigenvector basis. Furthermore the non-negativity of the $B_{i j}=A_{i j} w_{i j}$ matrix involves a unique, real, non-negative largest eigenvalue $\Lambda_{1}$. In the QMF approximation one can find $\lambda_{c}$ and $\rho(\lambda)$ around it by taking into account the principal eigenvector only. Using the linear superposition expansion of $\rho$ one can solve Eq. (1), which provides $\Lambda_{1}=1 / \lambda_{c}$, i.e., a stable $\rho>0$ (active) solution for $\lambda>\lambda_{c}$ and an inactive one for $\lambda \leqslant \lambda_{c}$. The order parameter near above $\lambda_{c}$ can be approximated via

$$
\rho(\lambda) \approx a_{1} \Delta+a_{2} \Delta^{2}+\cdots,
$$

where $\Delta=\lambda \Lambda_{1}-1 \ll 1$ and the coefficients,

$$
a_{j}=\sum_{i=1}^{N} f_{i}\left(\Lambda_{j}\right) /\left[N \sum_{i=1}^{N} f_{i}^{3}\left(\Lambda_{j}\right)\right],
$$

are functions of eigenvectors of the largest eigenvalues. This expression is exact if there is a gap between $\Lambda_{1}$ and $\Lambda_{2}$ [40].

It was proposed in [32] and tested on weighted BA models [28] that the localization of activity in the active steady state can be characterized by the inverse participation ratio (IPR), related to the eigenvector of the largest eigenvalue $f\left(\Lambda_{1}\right)$ as

$$
\operatorname{IPR}(N) \equiv \sum_{i=1}^{N} f_{i}^{4}(\Lambda)
$$

This quantity remains small: $\lim _{N \rightarrow \infty} \operatorname{IPR}(N)=0$ in case of homogeneous eigenvector components and takes the maximal value 1 if all activity is concentrated on a single node. I used the sparse matrix package OCTAVE [41] for generating and diagonalizing $B_{i j}$ and calculating $\operatorname{IPR}(N), \Lambda_{1}(N), a_{i}(N)$ for network sizes up to $N=200000$. In the numerical analysis I extrapolated and fitted the $\operatorname{IPR}(N)$ data assuming a power-law form,

$$
\operatorname{IPR}=\operatorname{IPR}(N)+X(1 / N)^{b},
$$

containing the free parameters: $X$ and $b$.

It was derived by [42], that the largest eigenvalue of $A_{i j}$ of general random graphs is determined by the maximum degree $k_{\text {max }}$,

$$
\Lambda_{1}(N)=(1+o(1)) \max \left\{\sqrt{k_{\max }},\left\langle k^{2}\right\rangle /\langle k\rangle\right\},
$$

where the $o(1)$ term tends to zero as the limiting values $\max \left\{\sqrt{k_{\max }},\left\langle k^{2}\right\rangle /\langle k\rangle\right\}$ diverge to infinity.

For the classical, random ER graph case, with finite connection probability: $p=\langle k\rangle / N$ we have $k_{\max }=\ln N / \ln \ln N$, therefore,

$$
\Lambda_{1}(N)=(1+o(1)) \max \{\sqrt{\ln N / \ln \ln N}, N p\} .
$$

Although in the $N \rightarrow \infty$ limit the first term dominates the maximum and predicts a divergence,

$$
\Lambda_{1}(N)=\sqrt{\ln N / \ln \ln N},
$$

this function grows so slowly, that even for extremely large sizes: $\Lambda_{1}\left(10^{9}\right)<2.7$ and practically one observes a constant value. Thus, for $\langle k\rangle \geqslant 3$ and for $N<2 \times 10^{5}$ graphs considered here the largest eigenvalue seems to tend to the finite value,

$$
\Lambda_{1}(N)=(1+o(1))\langle k\rangle,
$$

in agreement with the HMF theoretical value [4]:

$$
\lambda_{c}^{\mathrm{HMF}}=\langle k\rangle /\left\langle k^{2}\right\rangle,
$$

and with recent simulation results of Ref. [43].

For the random, unweighted SF networks, with power-law degree distribution $P(k) \propto k^{-3}$ the largest eigenvalue diverges and follows the finite size scaling law,

$$
\Lambda_{1}(N) \propto N^{1 / 4},
$$

deduced in [44]. Generally, in the numerical analysis of the QMF results least-squares fitting with the simple power-law form,

$$
1 / \Lambda_{1}=\lambda_{c}+Y(1 / N)^{c},
$$

was applied, but in the ER case the logarithmic convergence of the largest eigenvalue (8) has been tested.

\section{SIS MODEL SIMULATIONS}

Simulations of the SIS model were performed in such a way that in a given time step either a deactivation at site $i$, with probability: $1 /(1+\lambda)$ or activation of all neighboring inactive sites with probabilities, $w_{i j} \lambda /(1+\lambda) / N_{i}$, were attempted. Here $N_{i}$ is the number of neighboring, inactive sites, which was computed when the node $i$ was selected randomly. These reaction steps were iterated $N_{a}$ times, where $N_{a}$ is the number of active sites at time $t$. Following this system update, which selects each node once in average the time was incremented by $\Delta t=1$ Monte Carlo step (MSc). Throughout the paper 
the time is measured by MCs. The density of active sites $\rho(t)$ was calculated and stored at exponentially growing time steps: $t_{i}=1+1.08^{i}$. The system was initialized from a fully active state and the graph updates were repeated until $t<t_{\max }$ or in case of extinction of activity. The maximum simulation time depends on the system size, ranging from $t_{\max }=10^{7}$ for $N=10^{5}$ to $t_{\max }=10^{5}$ for $N=10^{6}$. To obtain good statistics the simulations have been repeated for $10^{2}-10^{4}$ independent graph realizations and $\rho(t)$ averaged over them.

To explore in more detail the decay of the density functions, I have computed effective decay exponents of the power laws $\rho(t) \propto t^{-\alpha}$, defined as the local slopes,

$$
\alpha_{\mathrm{eff}}(t)=-\frac{\ln \left[\rho(t) / \rho\left(t^{\prime}\right)\right]}{\ln \left(t / t^{\prime}\right)},
$$

where $t$ and $t^{\prime}$ was chosen in such a way that the discrete approximate of the derivative is sufficiently smooth.

\section{THE SIS MODEL ON ER GRAPHS}

In the Erdős-Rényi graph a giant connected component emerges for $\langle k\rangle \geqslant 1$ [45]. Above this phase transition point an arbitrarily large connected subgraph may exist and $d=\infty$. It has been conjectured [17,19] that in this case the epidemic spreading is too fast to let the formation of active RRs of size $l$ with the lifetime: $\tau \propto \exp (l)$, hence GP cannot occur. Contrary, for $\langle k\rangle<1$ the topological dimension is zero in the ER graph. It was also hypothesized [17,19] and shown by simulations of the $\mathrm{CP}$ [46], that in the fragmented phase strong rare-region effects and GP dynamics can be observed.

Now I investigate this hypothesis by simulations and with the help of the QMF method in case of the SIS model. Application of the QMF leads to the following results. In the percolating phase with $\langle k\rangle=4$ the $\operatorname{IPR}(N)$ value decays to zero as $\sim 1 / N$, indicating the disappearance of activity clustering in the steady state as shown on Fig. 1. This behavior is in agreement with the recent numerical results

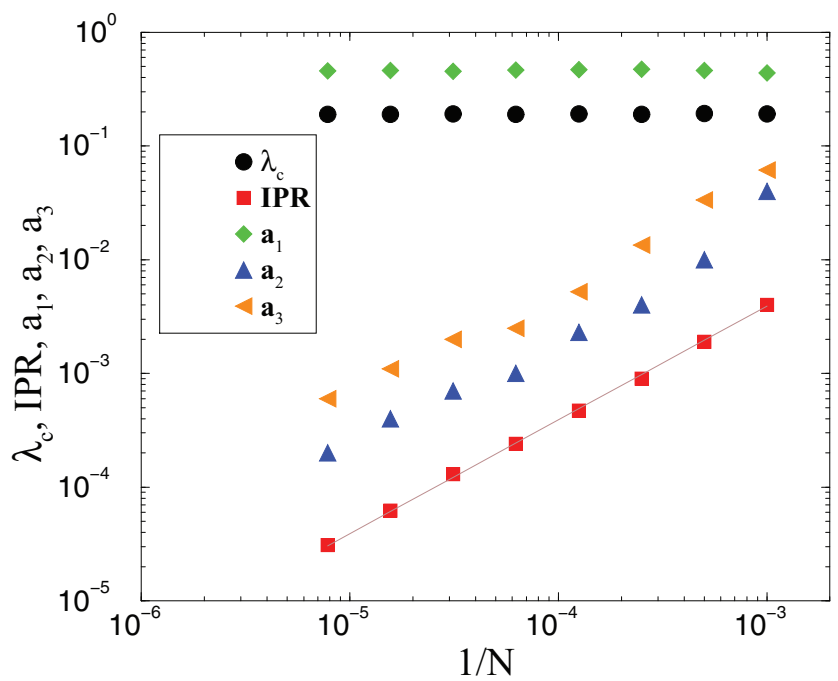

FIG. 1. (Color online) Finite size scaling of QMF results on the ER model with $\langle k\rangle=4$ for $N=1000,2000,4000, \ldots, 128000$. Bullets, $\lambda_{c}$; boxes, IPR; up-triangles, $a_{1}$; down-triangles, $a_{2}$; righttriangles, $a_{3}$. (Line) Least-squares fitting with the form $\sim 1 / N$.
TABLE I. Spectral QMF analysis results for SIS in different networks.

\begin{tabular}{lccrl}
\hline \hline Network & $1 / \Lambda_{1}$ & $c$ & \multicolumn{1}{c}{ IPR } & \multicolumn{1}{c}{$b$} \\
\hline ER-4 & $0.19(1)$ & $1.1(1)$ & $0.00003(5)$ & $1.1(1)$ \\
ER-03 & $0.01(1)$ & - & $0.22(2)$ & $0.9(2)$ \\
ER-4-E6 & $0.01(1)$ & - & $0.22(3)$ & $0.9(3)$ \\
ER-4-E1 & $0.30(3)$ & $0.34(1)$ & $0.01(1)$ & $0.93(9)$ \\
GBA2 & $0.001(2)$ & $0.18(5)$ & $0.28(5)$ & $0.5(1)$ \\
\hline \hline
\end{tabular}

of [47], obtained by diagonalizing adjacency matrices of ER graphs up to $N=10^{4}$. On the other hand $a_{1}$ is roughly constant, while $a_{2}$ and $a_{3}$ extrapolate to zero, suggesting a clean mean-field critical transition, characterized by $\beta=1$ leading order parameter exponent in agreement with our expectations. In the $N \rightarrow \infty$ limit extrapolation with the form (11) results in $\Lambda_{1}=5.23(3)$, which is larger than what a simulation should show for reasonable graph sizes $\left(N<10^{9}\right)$ : $\Lambda_{1}=4$ [see Eq. (9)]. The critical point estimate of QMF is: $\lambda_{c}=1 / \Lambda_{1}=0.191(1)$. Probably the application of pair, or higher level QMF, taking into account longer correlations of the order parameter would increase this value as in [48] for random regular networks. These results are summarized in the first line of Table I.

Extensive simulations for the more interesting, limiting case $\langle k\rangle=1$ have been performed for graphs with $N=10^{6}$ nodes. As Fig. 2 shows a mean-field type of phase transition appears at $\lambda_{c}=1.094(1)$, which is close to the the HMF value $\lambda_{c}^{\mathrm{HMF}}=1$.

In the fragmented phase, for $\langle k\rangle=0.3$ the IPR remains constant and tends to $0.22(2)$ (see Fig. 3) and the coefficients of the order parameter $\rho(\lambda)$ vanish as $a_{i} \propto 1 / N$. The epidemic threshold estimate $\lambda_{c}=1 / \Lambda_{1}$ decreases very slowly with $N$. The inset of Fig. 3 shows the slowly increasing $\Lambda_{1}$ approximated with the form (8). However, the density fluctuations of $\rho$ drive finite clusters, thus the whole system into the absorbing

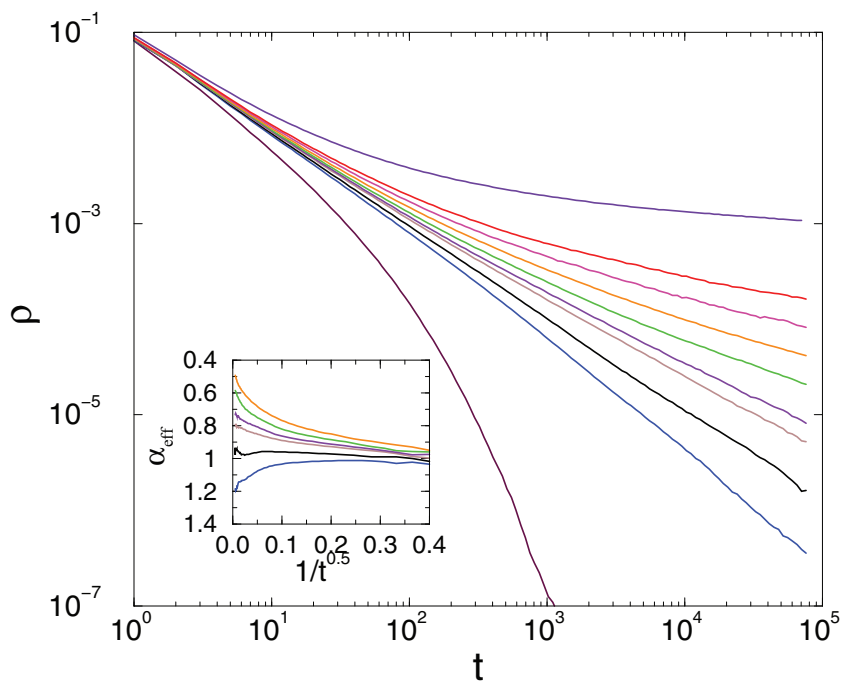

FIG. 2. (Color online) Density decay as a function of time for the SIS on ER graph with $\langle k\rangle=1$. Network size $N=10^{6}$. Different curves correspond to $\lambda=1.05,1.09,1.095,1.1,1.02,1.05,1.095$, $1.115,1.12,1.15$ (from bottom to top curves). (Inset) Effective exponents of the same data near the phase transition point. 


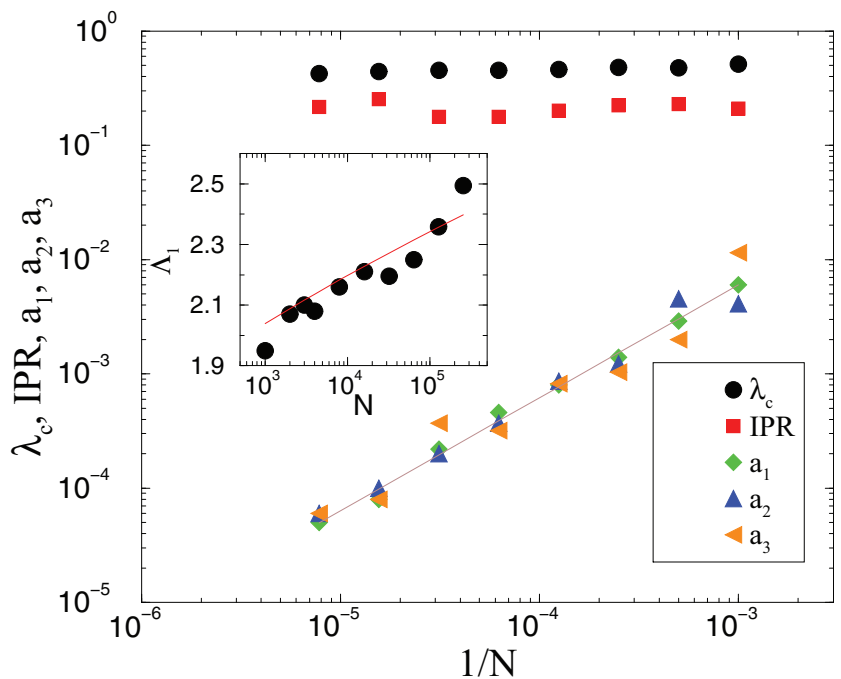

FIG. 3. (Color online) Finite size scaling of QMF results for ER graph with $\langle k\rangle=0.3 N=1000,2000,4000, \ldots 128000$. Bullets, $\lambda_{c}$; boxes, IPR; up-triangles, $a_{1}$; down-triangles, $a_{2}$; right-triangles, $a_{3}$. (Line) Least-squares fitting with a form $1 / N$. (Inset) $\Lambda_{1}(N)$ (bullets) and the form (8) (line).

state in the fragmented phase, which cannot be described by the QMF. Since a real GP singularity causes dynamical behavior even in the active phase the clustering behavior obtained by the $\mathrm{SD}$ decomposition predicts the existence of strong RR effects correctly. This underlines the capability of QMF to treat the effects of topological disorder well. The numerical results are summarized in the row "ER-03" of Table I.

The simulations confirm the existence of GP by showing an extended region of $\lambda$ dependent power laws of the density decay. As one can see in Fig. 4 for $\langle k\rangle=0.3$ the slopes of the curves on the log.-log. plot vary in wide range from $\simeq 1.34(1)$ at $\lambda=4$ to $\simeq 0.43$ (2) at $\lambda=20$. For higher $\lambda$ 's the decay curves

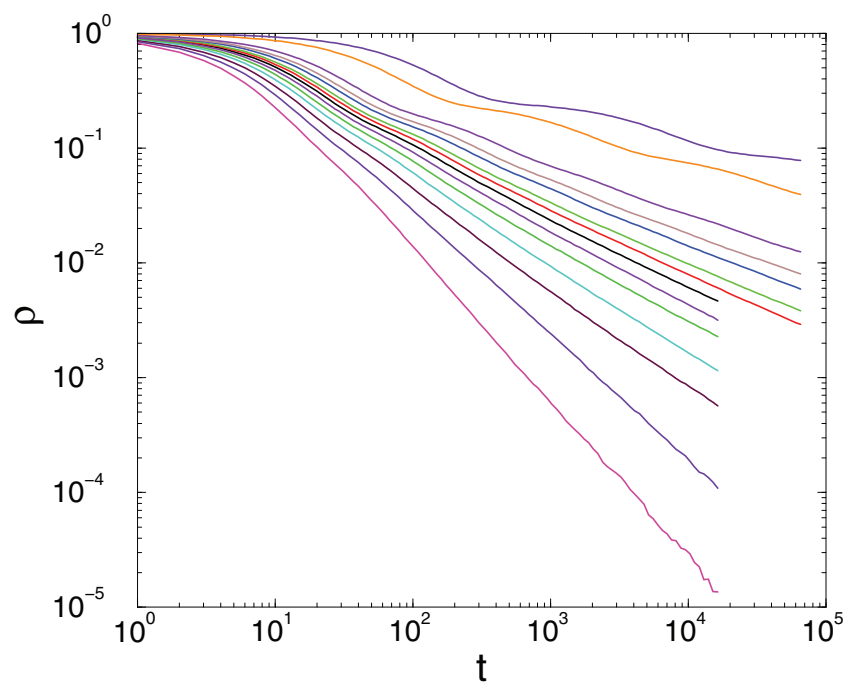

FIG. 4. (Color online) Density decay as a function of time in the SIS defined on ER graph with $\langle k\rangle=0.3$. Network size $N=10^{6}$. Different curves correspond to $\lambda=4,5,6,7,8,9,10,11,12,14,16$, 20, 50, 100 (from bottom to top curves).

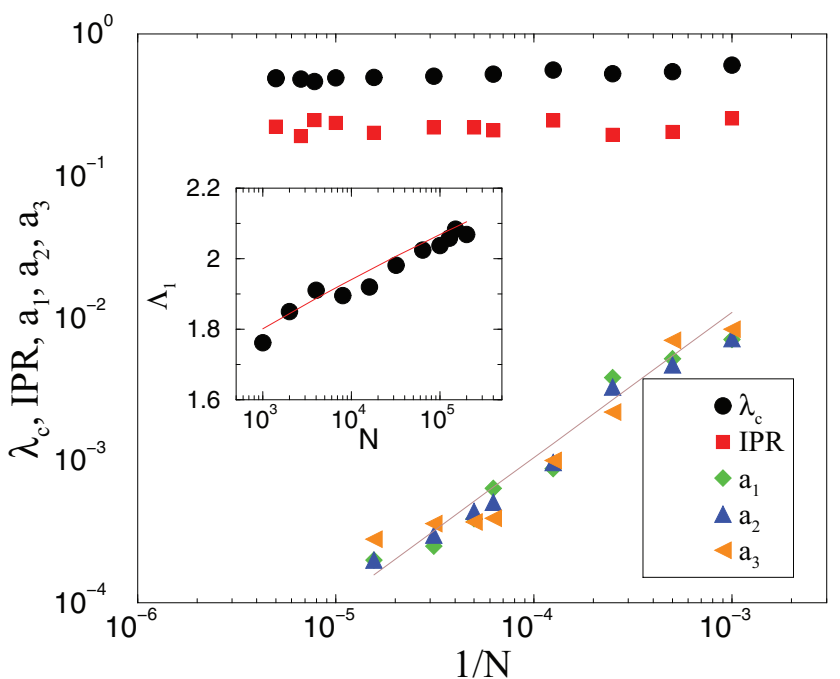

FIG. 5. (Color online) Finite size scaling of QMF results for the weighted ER graphs for $\langle k\rangle=4$, in the range of sizes $N=1000$, $2000 \ldots 200000$. Bullets, $\lambda_{c}$; boxes, IPR; up-triangles, $a_{1}$; downtriangles, $a_{2}$; right-triangles, $a_{3}$. (Line) Least-squares fitting with a $1 / N$. (Inset) $\Lambda_{1}(N)$ (bullets) and the form (8) (line).

show oscillations superimposed on the power law. This can be explained by the gradual deactivation of RRs in the fragments.

Finally, I have also studied the steady-state behavior by QMF on a weighted ER model introduced in [35]. This model was motivated by the face-to-face experiments analyzed in [36] with infection rates $\lambda w_{i j}$, proportional to the intensity of contacts, such that the quenched weights generate a $P(\omega)=$ $1 / \omega$ probability distribution function. In this model both the simulations, HMF and percolation approaches [35], suggest $\mathrm{RR}$ effects in the percolating phase of ER with $\langle k\rangle=4$. Weight factors are displaced on the graph edges,

$$
w_{i j}=\exp (-a r),
$$

where $r \in(0,1)$ is a uniformly distributed random variable and $a$ controls the strength of the disorder $w_{i j} \in\left(1 / e^{a}, 1\right)$ as in Ref. [35].

The QMF analysis for $a=6$ shows that this kind of disorder suppresses the infection rates very efficiently and one arrives at very similar results as for the fragmented case (see Fig. 5). The $\operatorname{IPR}(N)$ values are finite and constant, the coefficients $a_{i}$ decay as $\sim 1 / N$, and $\Lambda_{1}(N)$ follows the theoretical form (8) (see inset of Fig. 5). The $N \rightarrow \infty$ extrapolated values are summarized in the row "ER-4-6" of Table I. This means that due to the lower cutoff value of the weights the network is quasifragmented and the RR effects reported in [35] can be supported by the QMF method.

On the other hand, if the disorder is weak, characterized by $a=1$ a similar QMF analysis leads to the values shown in the row "ER-4-E1" of Table I. In this case $\Lambda_{1}(N)$ remains finite, while the $\operatorname{IPR}(N)$ converges to zero, meaning the lack of RR effects in agreement with the phase diagram shown in [35].

\section{THE SIS MODEL ON AGING BA GRAPHS}

In this section, I show the application of the QMF method to generalized BA tree networks (BAT) in which the spreading 


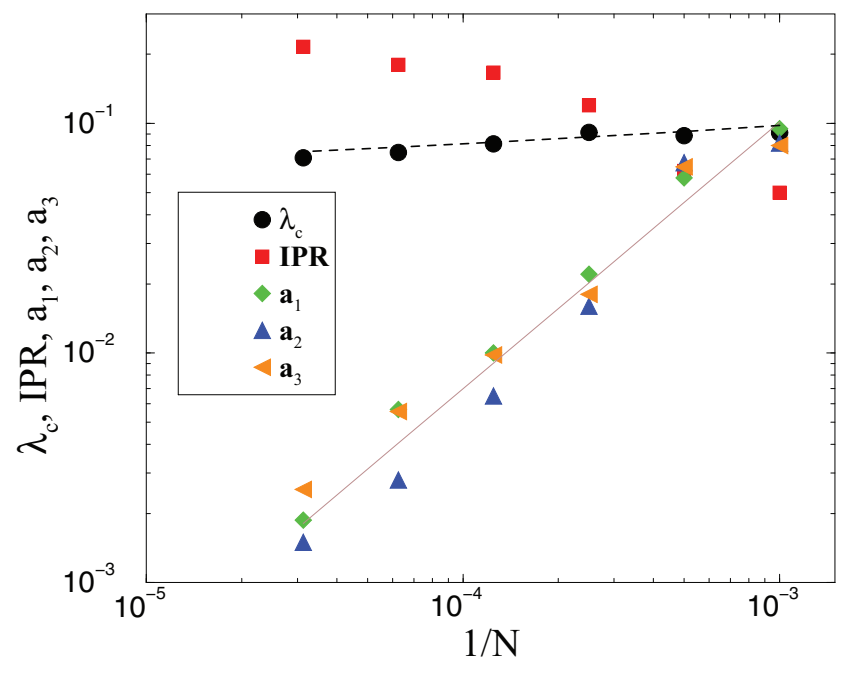

FIG. 6. (Color online) Finite size scaling of QMF SD results of the SIS on generalized BAT with $N=1000,2000,4000,8000$, 16000,32000 nodes. Bullets, $\lambda_{c}$; boxes, IPR; up-triangles, $a_{1}$; downtriangles, $a_{2}$; right-triangles, $a_{3}$. (Line) Least-squares fitting with the form $\sim 1 / N$.

behavior is weakened by preferential depletion of the links [39]. In Ref. [28] SIS models on top of BA graphs have been studied by QMF and extensive dynamical simulations. For these infinite topological dimensional networks no strong rareregion effects have been found, except when a dissortative weighting scheme was applied, which suppresses the hub-hub connections. This slows down the fast epidemic spreading in case of tree BA topologies.

Now I consider the SIS model on generalizations of BA types of networks [26]. The choice of this model is motivated by the fact that it allows one to construct tree structures in a very simple way, in contrast with other standard network

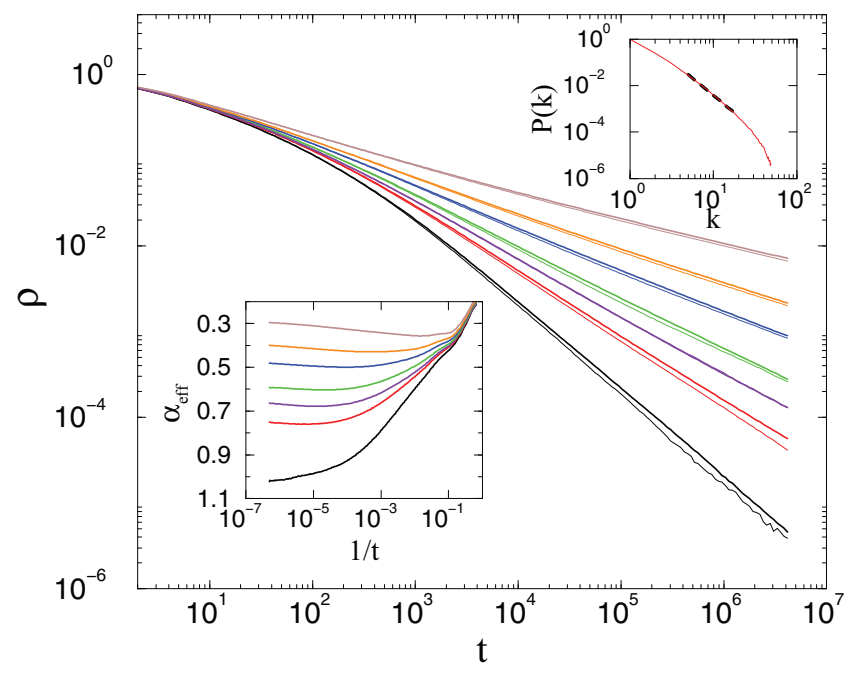

FIG. 7. (Color online) Density decay as a function of time for the SIS on BA graph with network sizes: $N=10^{5}$ (thin lines), $N=10^{6}$ (thick lines). Different curves correspond to $\lambda=2.4,2.45,2.47,2.5$, 2.55, 2.6, 2.7 (from bottom to top curves). (Left inset) Local slopes of the same data. (Right inset) Degree distribution of the aging BA graph for $N=4 \times 10^{6}$ nodes. generation models, e.g., [49]. BA is a growing network model in which, at each time step $s$, a new vertex (labeled $s$ ) with $m$ edges is added to the network and connected to an existing vertex $s^{\prime}$ of degree $k_{s^{\prime}}$ with probability $\Pi_{s \rightarrow s^{\prime}}=k_{s^{\prime}} / \sum_{s^{\prime \prime}<s} k_{s^{\prime \prime}}$. This process is iterated until reaching the desired network size $N$. The resulting network has an SF degree distribution $P(k) \simeq 2 m^{2} k^{-3}$; additionally, fixing $m=1$ leads to a strictly tree (loopless) topology.

Following the generation of BAT an aging procedure was applied on the network by gradually removing a fraction of randomly selected links connecting nodes $i$ and $j$ with the probability,

$$
q_{i, j}=\left(k_{i} k_{j}\right) / \sum_{i=1}^{N} k_{i} .
$$

The procedure was repeated until $\sum_{i} k_{i=1}^{N} \geqslant 1.6 N$, i.e., $20 \%$ of the original links of the graph were removed. As a consequence the original scale-free distribution $P(k) \propto k^{-3}$ gets an exponential cutoff as shown in the right inset of Fig. 7. This is quite similar to what one observes in most empirical degree distributions. Here the topological dimension of the graph becomes zero, because this edge dilution removes the possibility of infinitely large components. On the other hand, an edge dilution with nonlinear depreciation probability as in [50] can keep the power law in $P(k)$ and its dynamical behavior should be studied further.

The SD analysis of this model (GBA2) gives IPR results with strong clustering in the $N \rightarrow \infty$ limit, thus it suggests rare-region effects (see Fig. 6). While the IPR converges to $0.18(5)$ the largest eigenvalue increases and the coefficients $a_{i}$ decrease as $\sim 1 / N$. A power-law fit for the critical point estimate results in $\lambda_{c}=1 / \Lambda_{1}=0.001+0.17(1 / N)^{0.18}$, which is similar to the behavior found in the case of SIS on BA graphs [28]. The finite size scaling exponent $c$ is close to the value predicted by analytical considerations [see Eq. (11)].

Simulations show clear $\lambda$-dependent, power-law density decays (see Fig. 7). The effective decay exponents saturate to constant values in the large time limit (left inset of Fig. 7) albeit some (log.) correction is possible. Furthermore, the results do not seem to depend on the size of the networks used. These are well-known indications of Griffiths Phases.

\section{CONCLUSIONS}

In conclusion the suitability of the QMF method for detecting rare-region effects has been demonstrated on different complex networks. Comparison with dynamical simulations confirms that the clustering behavior in the steady state is related to the occurrence of generic slow behavior and Griffiths Phases. The localization transition in the eigenvector, characterized by the IPR, connects these results to the theory of random matrices [51,52] and metal-insulator transitions [53]. Finite size scaling and asymptotic values of the largest eigenvalue of the adjacency matrix agree with the theoretical predictions for the SIS model defined on top of ER and BA networks. In particular, the slow logarithmic decrease of the threshold value for random networks is studied. In the fragmented phase QMF results in inhomogeneous, clustered principal eigenvector. However, it cannot describe well the 
inactive phase, because it neglects dynamical fluctuations, which in a real system drive all finite clusters to extinction in finite times. Still, it predicts correctly the relevancy of topological disorder in agreement with the GP found by the simulations. In the percolating phase of ER graph the QMF method results in the irrelevancy of network heterogeneity on the SIS scaling, providing the expected mean-field critical behavior. This has been confirmed by dynamical simulations at $\langle k\rangle=1$.

The methodology has also been applied to two more interesting cases, where spectral decomposition of weighted adjacency matrices is performed. I considered two important cases, where GP behavior could be expected. In case of a quenched weight scheme, capable of describing phenomenologically observed face-to-face inter-event distribution the QMF supports recent numerical simulation results. However, it is more likely that the dynamical power-law behavior is restricted to finite times, because in an infinite dimensional graph arbitrarily large (dimensional) subspaces may survive an extinction process and provide finite contribution to the density. For weak disorders it predicts the lack of GP on the percolating ER model. It has also been shown that QMF describes well the GP behavior of SIS on a Barabási-Albert type model with aging nodes, i.e., when links are diluted by a preferential detachment rule. This is confirmed by dynamical simulations, exhibiting nonuniversal power laws, with negligible size dependencies.

Finite size scaling of the QMF results also predict the disappearance of the largest three amplitudes $\left(a_{i}\right)$ of the order parameter as $\sim 1 / N$, except for the nonclustering ER cases, when the leading order one $\left(a_{1}\right)$ remains constant, meaning a linear mean-field exponent with negligible corrections. These results corroborate a previous conjecture [17] according to which slow dynamics in the thermodynamic limit can be found in network models with finite topological dimensions. Application of the present method to real networks, where large-scale modularity is an ubiquitous property [54] would reveal interesting consequences on the dynamics of processes evolving on them.

\section{ACKNOWLEDGMENTS}

I thank R. Juhász and R. Pastor-Satorras for useful comments. Support from the Hungarian research fund OTKA (Grant No. T77629) and the European Social Fund through project FuturICT.hu (Grant No. TAMOP-4.2.2.C-11/1/KONV2012-0013) is acknowledged.
[1] S. N. Dorogovtsev, A. V. Goltsev, and J. F. F. Mendes, Rev. Mod. Phys. 80, 1275 (2008).

[2] A. Barrat, M. Barthélemy, and A. Vespignani, Dynamical Processes on Complex Networks (Cambridge University Press, Cambridge, 2008).

[3] S. Johnson, J. J. Torres, and J. Marro, PLoS ONE 8, e50276 (2013).

[4] R. Pastor-Satorras and A. Vespignani, Phys. Rev. Lett. 86, 3200 (2001).

[5] R. Pastor-Satorras and A. Vespignani, Evolution and Structure of the Internet: A Statistical Physics Approach (Cambridge University Press, Cambridge, 2004).

[6] A. Haimovici, E. Tagliazucchi, P. Balenzuela, and D. R. Chialvo, Phys. Rev. Lett. 110, 178101 (2013).

[7] M. Karsai, M. Kivela, R. K. Pan, K. Kaski, J. Kertesz, A. L. Barabasi, and J. Saramaki, Phys. Rev. E 83, 025102(R) (2011).

[8] J. Marro and R. Dickman, Nonequilibrium Phase Transitions in Lattice Models (Cambridge University Press, Cambridge, 1999).

[9] G. Ódor, Rev. Mod. Phys. 76, 663 (2004).

[10] G. Ódor, Universality in Nonequilibrium Lattice Systems (World Scientific, Singapore, 2008).

[11] R. B. Griffiths, Phys. Rev. Lett. 23, 17 (1969).

[12] T. Vojta, J. Phys. A: Math. Gen. 39, R143 (2006).

[13] A. Amir, Y. Oreg, and Y. Imry, Phys. Rev. Lett. 105, 070601 (2010).

[14] C. Monthus and T. Garel, J. Phys. A: Math. Theor. 44, 085001 (2011).

[15] I. A. Kovács and F. Iglói, J. Phys.: Condens. Matter 23, 404204 (2011).

[16] R. Juhász and I. A. Kovács, J. Stat. Mech. (2013) P06003.

[17] M. A. Muñoz, R. Juhász, C. Castellano, and G. Ódor, Phys. Rev. Lett. 105, 128701 (2010).
[18] G. Ódor, R. Juhasz, C. Castellano, and M. A. Munoz, in Nonequilibrium Statistical Physics Today, edited by P. L. Garrido, J. Marro, and F. de los Santos, Vol. 1332 (AIP, Melville, 2011), pp. 172-178.

[19] R. Juhász, G. Ódor, C. Castellano, and M. A. Muñoz, Phys. Rev. E 85, 066125 (2012).

[20] P. Erdos and A. Rényi, A. Publ. Math. 6, 290 (1959).

[21] T. E. Harris, Ann. Prob. 2, 969 (1974).

[22] T. M. Liggett, Interacting Particle Systems (Springer-Verlag, New York, 1985).

[23] M. Aizenman and C. M. Newman, Commun. Math. Phys. 107, 611 (1986).

[24] R. Juhász and G. Ódor, Phys. Rev. E 80, 041123 (2009).

[25] R. Juhász, Phys. Rev. E 78, 066106 (2008).

[26] A.-L. Barabási and R. Albert, Science 286, 509 (1999).

[27] G. Odor and R. Pastor-Satorras, Phys. Rev. E 86, 026117 (2012).

[28] G. Odor, Phys. Rev. E 87, 042132 (2013).

[29] R. M. Anderson and R. M. May, Infectious Diseases in Humans (Oxford University Press, Oxford, 1992).

[30] M. Boguñá, C. Castellano, and R. Pastor-Satorras, Phys. Rev. E 79, 036110 (2009).

[31] S. C. Ferreira, C. Castellano, and R. Pastor-Satorras, Phys. Rev. E 86, 041125 (2012).

[32] A. V. Goltsev, S. N. Dorogovtsev, J. G. Oliveira, and J. F. F. Mendes, Phys. Rev. Lett. 109, 128702 (2012).

[33] D. Chakrabarti, Y. Wang, C. Wang, J. Leskovec, and C. Faloutsos, ACM Trans. Inf. Syst. Secur. 10, 1 (2008).

[34] P. Van Mieghem, J. Omic, and R. Kooij, IEEE ACM T. Network. 17, 1 (2009).

[35] C. Buono, F. Vazquez, P. A. Macri, and L. A. Braunstein, Phys. Rev. E 88, 022813 (2013).

[36] C. Cattuto, W. V. den Broeck, A. Barrat, V. Colizza, J.-F. Pinton, and A. Vespignani, PLoS ONE 5, e11596 (2010). 
[37] Y.-Ho Eom and A. Fortunato, Plos ONE 6, e24296 (2011).

[38] S. N. Dorogovtsev and J. F. F. Mendes, Phys. Rev. E 62, 1842 (2000).

[39] C. M. Schneider, L. de Arcangelis, and H. J. Herrmann, Europhys. Lett. 95, 16005 (2011).

[40] P. Van Mieghem, Eur. Phys. Lett. 97, 48004 (2012).

[41] http://www.gnu.org/software/octave.

[42] F. Chung, L. Lu, and V. Vu, Proc. Natl. Acad. Sci. USA 100, 6313 (2003).

[43] M. Boguña, C. Castellano, and R. Pastor-Satorras, arXiv:1305.4819.

[44] A. Halu, S. Garnerone, A. Vezzani, and G. Bianconi, Phys. Rev. E 87, 022104 (2013).
[45] M. Molloy and B. A. Reed, Random Struct. Algor. 6, 161 (1995). [46] G. Ódor, EPJ Web of Conferences 44, 04005 (2013).

[47] F. Slanina, Eur. Phys. J. B 85, 361 (2012).

[48] A. S. Mata and S. C. Ferreira, arXiv:1305.5153.

[49] M. Catanzaro, M. Boguñá, and R. Pastor-Satorras, Phys. Rev. E 71, 027103 (2005).

[50] A. A. Moreira, J. S. Andrade, H. J. Herrmann, and J. O. Indekeu, Phys. Rev. Lett. 102, 018701 (2009).

[51] B. Bollobás, Random Graphs (Academic Press, London, 1985).

[52] G. J. Rodgers and A. J. Bray, Phys. Rev. B 37, 3557 (1988).

[53] P. W. Anderson, Phys. Rev. 109, 1492 (1958).

[54] M. E. J. Newman, Nat. Phys. 8, 25 (2012). 